\title{
Stroke Intervention: Geographic Disparities in Acute Stroke Care and the Role of Interventional Cardiology
}

We have featured as our Editor's Pick for Interventional Cardiology 8.1 this discussion by White et al. of disparities in acute stroke care delivery due to regional service differences. Randomised studies in acute ischaemic stroke have demonstrated the superiority of mechanical thrombectomy over systemic EDITOR'S thrombolysis in achieving reperfusion of large vessel occlusion. For more than four decades, interventional cardiologists have accumulated experience in treating acute coronary syndromes, and integration into stroke teams may help to achieve timely reperfusion and solve the disparities in access to specialised stroke centres, as shown in this article.

\section{Authors:}

Disclosure:

Received:

Accepted:

Keywords:

Citation:
*Christopher J. White, Jose D. Tafur, Tamunoinemi Bob-Manuel, Marloe Prince, Rajan A.G. Patel, J. Stephen Jenkins, Tyrone J. Collins, Stephen R. Ramee

Ochsner Medical Center, New Orleans, Louisiana, USA

*Correspondence to cwhite@ochsner.org

The authors have declared no conflicts of interest.

13.01.20

19.02.20

Acute stroke intervention, carotid stent, disparities in care, interventional cardiology, mechanical thrombectomy (MT).

EMJ Int Cardiol. 2020;DOI/10.33590/emjintcardiol/20-00009

\section{Abstract}

Stroke is the second-leading cause of death and a major cause of disability worldwide. The majority of strokes are ischaemic, and effective therapy to achieve reperfusion includes intravenous thrombolysis and, for proximal large vessel occlusion strokes, endovascular mechanical thrombectomy (MT). There has been a paradigm shift in acute stroke care, driven by a series of randomised controlled trials demonstrating that timely reperfusion with MT results in superior outcomes compared to intravenous thrombolysis in patients with large vessel occlusion strokes. There are significant geographic disparities in delivering acute stroke care because of the maldistribution of neurointerventional specialists. There are now several case series demonstrating the feasibility and safety of first medical contact MT by carotid stent-capable interventional cardiologists and noninvasive neurologists working on stroke teams, which is a solution to the uneven distribution of neurointerventionalists and allows stroke interventions to be delivered in local communities. 


\section{INTRODUCTION}

Stroke is the second-leading cause of death and a major cause of disability worldwide. Its incidence is increasing because the population is ageing. ' Stroke rates in the young and in middle-aged adults are not decreasing and may even be increasing, likely because of the growing prevalence of atherosclerotic risk factors, including obesity and diabetes. In highincome countries, improvements in prevention, acute treatment, and neurorehabilitation have led to a substantial reduction in the burden of stroke. However, significant geographic disparities remain in delivering care for acute stroke because of the uneven distribution of neurointerventional specialists.

The causes of stroke include haemorrhage, thrombosis, and embolus. Embolic strokes may be from artery to artery or from a heart chamber (left atrium or ventricle) to an artery, particularly in patients with atrial fibrillation. A major tenet for the treatment of ischaemic stroke is that 'time is brain', and that earlier reperfusion is associated with better outcomes.

The extent of ischaemic brain injury is determined by the time from the onset of symptoms to reperfusion and is significantly affected by the presence of collateral circulation, including an intact Circle of Willis. The penumbra is the region of the brain surrounding the infarct area, where the blood supply is reduced but viability is maintained due to collateral flow. The viability of the penumbra depends on the severity and duration of ischaemia. If blood flow is rapidly restored, ischaemic brain tissue can be salvaged.

Treatment goals include preventing or limiting the mortality and morbidity of the acute event, and preventing recurrent events. The majority of strokes are ischaemic ${ }^{2}$ and effective therapy to achieve reperfusion includes intravenous thrombolysis and, for proximal large vessel occlusion (LVO) strokes, endovascular mechanical thrombectomy $(\mathrm{MT})^{3}$

\section{STROKE THERAPY}

Although rapid initiation of intravenous tissuetype plasminogen activator (IV tPA) with a doorto-needle time of less than 60 minGet With the Guidelines ${ }^{\circledR}$-Stroke is important for a good outcome, the American Heart Association's $(\mathrm{AHA})$ Get With the Guidelines ${ }^{\circledR}$ - Stroke national registry reported that fewer than one in three stroke patients are treated within $60 \mathrm{~min}$ of arrival at the hospital. ${ }^{3,4}$ In the USA, a national heart attack quality initiative led interventional cardiologists to achieve dramatic reductions in door-to-balloon times for ST elevation myocardial infarctions; ${ }^{5}$ however, acute stroke therapy remains without a workable national strategy to provide timely reperfusion.

Currently, there is no time-to-reperfusion goal for door-to-treatment time in the USA and it is not standard of care for stroke therapy as it is for heart attacks. ${ }^{6}$
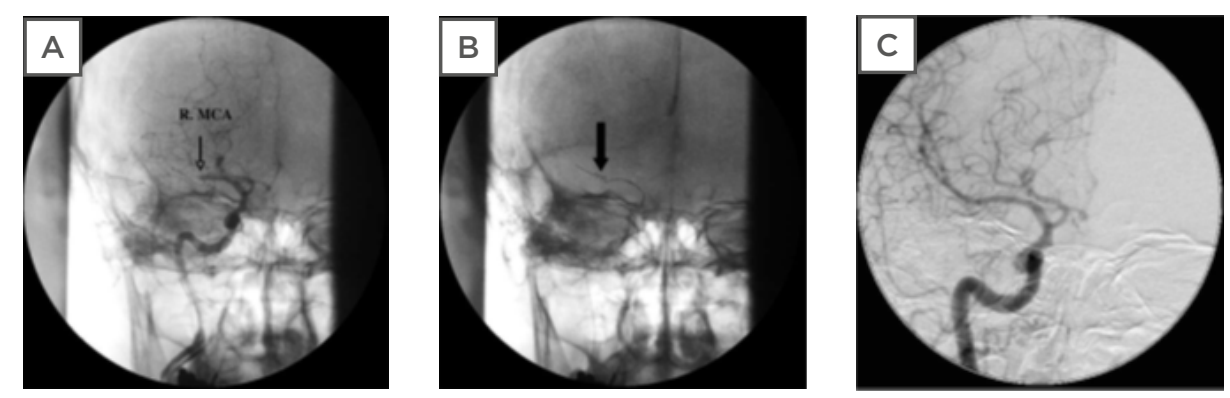

Figure 1: A) Patient with atrial fibrillation had an acute right middle cerebral artery occlusion; B) placement of the thrombectomy catheter (arrow) in the right middle cerebral artery; C) final result after thrombectomy of the right middle cerebral artery.

Adapted from Topol, Teirstein, 2019.12 
Table 1: Skills needed to care for stroke patients.

\begin{tabular}{|l|l|l|}
\hline Skill & Specialty & Description \\
\hline Cognitive & Neurology & $\begin{array}{l}\text { Knowledge of the natural history } \\
\text { and pathophysiology of stroke, } \\
\text { diagnostic methods, and treatment } \\
\text { options. }\end{array}$ \\
\hline Technical & IC, IR, NI, VS, VM & $\begin{array}{l}\text { Competence to perform } \\
\text { cerebrovascular angiography and } \\
\text { interventions (e.g., vascular access, } \\
\text { carotid stenting, MT). }\end{array}$ \\
\hline Clinical & Neurology, NCC & $\begin{array}{l}\text { The ability to care for acute stroke } \\
\text { patients including managing } \\
\text { complications, interpretation of } \\
\text { diagnostic tests, hospital admitting } \\
\text { privileges, and the ability to assess } \\
\text { the risk to benefit ratio for treatment } \\
\text { options. }\end{array}$ \\
\hline
\end{tabular}

IC: interventional cardiology; IR: interventional radiology; MT: mechanical thrombectomy; NCC: neurocritical care (anaesthesia, neurology, critical care); NI: neurointerventional (neuroradiology, neurosurgery, interventional neurology); VM: vascular medicine; VS: vascular surgery.

Unfortunately, because of a variety of issues including delayed time to presentation, lack of availability of rural ( $>1$ hour by ground transportation) endovascular stroke programmes, and a lack of national focus on time-to-treatment, many patients with ischaemic stroke in the USA do not receive timely reperfusion therapy. ${ }^{7}$

There has been a paradigm shift in acute stroke care, driven by a series of randomised controlled trials demonstrating that timely reperfusion with MT results in superior outcomes compared to IV tPA in patients with LVO (Figure 1). ${ }^{8-12}$

There was a consistent favourable clinical outcome (defined as a modified Rankin Scale [mRS] of $\leq 2$ at 90 days) for MT across all studies, with greater benefit seen with earlier intervention. When penumbral imaging with CT perfusion was used for patient selection, the advantage for MT was even more pronounced. Importantly, the use of MT added no additional risk of intracranial haemorrhage over standard management with IV tPA.

Although MT was associated with higher costs, it also resulted in better patient outcomes. Costeffectiveness studies revealed that MT adds value when a standard threshold of $\$ 50,000$ per quality-adjusted life year gained is adopted..$^{13}$

In the USA, 10\% of first-time ischaemic strokes have LVO and are potential candidates for MT. Minimising the delay to reperfusion is the key to optimising quality-of-life outcomes. It is estimated that every $10 \mathrm{~min}$ of delayed care reduces a patient's disability-free lifetime by approximately 40 days and reduces the net monetary benefit of MT by approximately $\$ 10,000 . .^{14}$

\section{GEOGRAPHIC DISPARITIES IN STROKE CARE}

There is a raging 'turf battle' between neurointerventionalists and non-neuroscience trained physicians who are capable of performing MT over who should be treating LVO acute strokes. ${ }^{15-17}$ Neurointerventionalists are predominantly clustered in urban, academic medical centres because their routine day-today work is facilitated by a centralised referral model. ${ }^{18,19}$ Endovascular stroke care, however, is time-sensitive, and many patients do not live close enough to the stroke centres to receive timely care. In the USA, only $50 \%$ of the 
population has $\leq 1$-hour access by ground transport to MT stroke treatment centres. In California, USA, only $39 \%$ of acute stroke patients live within 1 hour of hospitals performing 10 or more stroke interventions per year. ${ }^{20}$

\section{POTENTIAL SOLUTIONS}

One solution proposed by the neuro interventional community is the 'hub-and-spoke model' for transfer of stroke patients between hospitals. They suggest developing a national triage system to rapidly transfer stroke patients to highly specialised stroke centres. However, there is evidence that even a well-developed huband-spoke model is not an optimal solution for acute stroke therapy. An experienced European regional stroke centre initiated MT in 295 of 324 (91.0\%) patients directly admitted to the hub hospital, but initiated MT in only 63 of 91 (69.2\%) patients transferred to the hub from a regional hospital $(p<0.001) .{ }^{21}$ One quarter (24.2\%) of patients transferred became ineligible for MT during transfer. There was a four-fold improvement in the odds for a good clinical outcome in transferred patients who received
MT compared to those who became ineligible during transfer. The odds of a transferred patient receiving $\mathrm{MT}$ decreased by $2.5 \%$ for every minute of transfer time. The authors concluded that stroke intervention should be provided at first hospital contact whenever possible.

It has been suggested that training more neurointerventionalists would allow them to be deployed in rural areas to serve the needs of patients living more than 1 hour from stroke centres. Training more neurointerventionalists does not appear to be a practical solution as the volume of elective, daytime intracranial work in nonurban communities simply does not provide enough cases to support the additional neurointerventionalists needed to treat acute strokes or to maintain their skills. This uneven distribution of neurointerventionalists is manifested by efforts to reduce the number of their trainees because of oversupply. ${ }^{22,23}$

There has been a suggestion to offer practising non-invasive neurologists neurointerventional training whilst working in order to serve nonurban communities. ${ }^{24}$

Table 2: Results of interventional cardiology case series and neurointerventional randomised trials.

\begin{tabular}{|c|c|c|c|c|c|c|c|}
\hline $\begin{array}{l}\text { Author/ } \\
\text { Study }\end{array}$ & $N$ & $\begin{array}{l}\text { Baseline } \\
\text { NIHSS }\end{array}$ & $\begin{array}{l}\text { 30-90-day } \\
\text { mortality } \\
(\%)\end{array}$ & $\begin{array}{l}\text { mRS }<290 \\
\text { Day (\%) }\end{array}$ & $\mathrm{ICH}(\%)$ & $\begin{array}{l}\text { TICI flow } \\
2 b / 3 \text { (\%) }\end{array}$ & DTB (min) \\
\hline $\begin{array}{l}\text { Htyte et al., }{ }^{26} \\
2014\end{array}$ & 54 & 15 & 16.0 & 45.0 & 7.4 & 78 & 152 \\
\hline $\begin{array}{l}\text { Widimsky et } \\
\text { al.,27 } 2015\end{array}$ & 84 & 18 & 11.0 & 36.0 & 3.6 & 72 & 205 \\
\hline $\begin{array}{l}\text { Hornung et } \\
\text { al., } 282019\end{array}$ & 70 & 17 & 18.0 & 61.0 & 7.1 & 93 & 94 \\
\hline $\begin{array}{l}\text { Guidera et } \\
\text { al., }{ }^{29} 2018\end{array}$ & 40 & 19 & 15.0 & 55.0 & 15.0 & 80 & 108 \\
\hline IC (mean) & & 17 & 15.0 & 49.0 & 8.0 & 81 & 140 \\
\hline Mr Clean ${ }^{8}$ & 233 & 17 & 18.9 & 32.6 & 7.7 & 59 & 260 \\
\hline Escape $^{9}$ & 165 & 16 & 10.4 & 53.0 & 3.6 & 72 & 241 \\
\hline Revascat ${ }^{11}$ & 103 & 17 & 18.4 & 43.7 & 1.9 & 66 & 355 \\
\hline Swift Prime $e^{10}$ & 98 & 17 & 9.0 & 60.0 & 0.0 & 88 & 252 \\
\hline $\mathrm{NI}$ (mean) & & 17 & 14 & 47 & 3 & 71 & 277 \\
\hline
\end{tabular}

DTB: door-to-balloon (device) time; IC: interventional cardiology trials; ICH: intracranial haemorrhage; mRS: modified Rankin Scale; NI: neurointerventional trials; NIHSS: National Institute of Health Stroke Scale; TICI: thrombolysis in cerebral infarction flow grade. 
The possibility of training a practising non-invasive neurologist to perform stroke interventions is challenging, if not unrealistic. Moreover, it is doubtful that there is a sufficient pool of non-invasive neurologists to train, given the small number of practising stroke neurologists outside of academic medical centres.

A multidisciplinary group performed a 'needs assessment' for stroke intervention, with representatives from neurology, cardiology, vascular medicine, radiology, and neurosurgery disciplines. The group concluded: "We suggest taking what has been learned in treating ST elevation myocardial infarctions and apply it to 'brain attacks' utilising a multidisciplinary approach" (Table 1). ${ }^{25}$ This option would take advantage of currently available communitybased, carotid artery stent-capable interventional cardiologists with a basic knowledge of the intracranial circulation, paired with noninvasive neurologists and radiologists capable of interpreting CT images, and nurses capable of caring for stroke patients, to provide timely reperfusion therapy at first medical contact. The neurology support and guidance could be offered via telemedicine.

In community hospitals without stroke neurologists, or those with a single neurologist for whom being the on-call doctor would be too burdensome, telemedicine support from a stroke neurologist at a remote centre would be a solution to guide patient selection and management alongside the interventional cardiologist. Reperfusion therapy at first medical contact would expedite early treatment and, if needed, a less time-sensitive transfer to the stroke centre could be performed.

\section{CURRENT EVIDENCE}

There are several reports demonstrating the feasibility of offering safe and effective first medical contact stroke therapy by stroke teams consisting of carotid stent-capable interventional cardiologists and non-invasive neurologists (Table 2). ${ }^{26-29}$

Htyte et al. ${ }^{26}$ compared outcomes of interventional cardiologists versus neurointerventionalists in 124 consecutive acute stroke patients who received MT between 2006 and 2012.26 The on-call interventional team (interventional cardiologists versus neurointerventionalists) rotated responsibility for stroke calls. The interventional cardiologists had extensive carotid stenting experience and teamed with a non-invasive stroke neurologist who was responsible for pre- and post-management decisions. Interventional cardiologists treated 58 of 124 (47\%) patients and neurointerventionalists treated 66 of 124 (53\%) patients. There were no significant differences between the two groups in age, baseline National Institute of Health Stroke Scale (NIHSS), 30-day mortality, or a good clinical outcome $(\mathrm{mRS} \leq 2)$ at 90 days (Table 2$)$.

Interventional cardiologists from three European centres without neurointerventional services treated 84 consecutive acute stroke patients (NIHSS 26 ) with MT in partnership with noninvasive neurologists. ${ }^{27}$ Angiographic success (Thrombolysis in Cerebral Infarction [ $\mathrm{TICl}$ ] Grade $2 \mathrm{~b}$ or 3 ) was achieved in $72 \%$ of patients. A good neurological outcome ( $\mathrm{mRS} \leq 2$ at 90 days) was achieved in $42 \%$ (35 of 84 ) of patients, with 24 patients (29\%) discharged directly home. In patients treated within 3 hours of stroke onset, a good neurological outcome (mRS $\leq 2)$ was obtained in 54\% compared to $31 \%(p=0.031)$ in patients treated later than 3 hours. These acute stroke interventions by interventional cardiologists achieved results comparable to those from neurointerventional centres, suggesting that where local neurointerventional services are unavailable, emergent revascularisation by a carotid stent-capable interventional cardiologist, in partnership with a neurologist, is a reasonable option.

A multidisciplinary group from a community hospital located in Doylestown, Pennsylvania, USA ( $\geq 1$ hour from the nearest stroke centre in Philadelphia, Pennsylvania, USA), without the services of a neurointerventionalist, determined that local acute stroke care was needed. ${ }^{29}$ They formed a stroke team with carotid stent-capable interventional cardiologists, non-invasive neurologists, and a neuroradiology technician with interventional stroke experience. They treated 40 LVO stroke patients with MT after the neurologist reviewed their clinical and imaging data. The median NIHSS on presentation 
was 19 (severe disability). The average time from presentation in the emergency department to MT was $108 \mathrm{~min}$. $\mathrm{TICl}$ Grade 2b or 3 flow was achieved in $80 \%$ (32 of 40 ). Symptomatic intracranial haemorrhage occurred in $15 \%$ (6 of 40), with an in-hospital mortality of $13 \%$ (5 of 40 ). At 90 days, $55 \%$ of these severely affected patients were functioning independently ( $\mathrm{mRS} \leq 2$ ).

A recently published report from Frankfurt, Germany, reported the experience of a carotid stent-capable interventional cardiology group working with stroke neurologists to treat 70 consecutive LVO ischaemic strokes. ${ }^{28}$ The majority (90\%) of patients were admitted from their emergency department and $10 \%$ were transferred from local hospitals. On presentation to the emergency department, the average NIHSS was 17. The mean door-to-MT time was 94 min. Successful reperfusion $(\mathrm{TICl} 2 \mathrm{~b}$ or 3 flow) was achieved in 90\% (65 of 70), with a 30 -day mortality of $18 \%$ (13 of 70 ). At 90 days, $61 \%$ of their patients were functioning independently ( $\mathrm{mRS} \leq 2$ ).

These two recent reports from hospitals without neurointerventional availability, one from the USA and one from Germany, add to the growing body of evidence that carotid stent-capable interventional cardiologists can achieve excellent outcomes for acute stroke intervention in partnership with non-invasive neurologists. ${ }^{28,29}$

\section{SUMMARY}

There are now convincing data that carotid stentcapable interventional cardiologists, working in multidisciplinary stroke teams with neurology guidance, can achieve results in LVO acute stroke patients comparable to the landmark neurointerventional-driven MT trials (Table 2). This is even more remarkable given that randomised trials have strict selection criteria that exclude high-risk patients, while the interventional cardiology case series are real-world patients that include higher risk patients.

In major metropolitan medical centres, acute stroke intervention should be performed by neurointerventionalists. Given that a large percentage of acute stroke patients live long distances from these medical centres, and that time-to-reperfusion is critical to achieving good outcomes, a patient-centred solution that brings stroke care to these geographically disadvantaged patients is needed. Encouraging carotid stent-capable interventionalists to provide that care seems reasonable, and the safety and efficacy of this strategy is supported by a growing evidence base.

\section{References}

1. Katan M, Luft A. Global burden of stroke. Semin Neurol. 2018;38(2):20811.

2. Pencina $\mathrm{MJ}$ et al. Predicting the 30-year risk of cardiovascular disease: the framingham heart study. Circulation. 2009;119(24):3078-84.

3. Powers WJ et al. 2015 American Heart Association/American Stroke Association focused update of the 2013 guidelines for the early management of patients with acute ischemic stroke regarding endovascular treatment. Stroke. 2015;46(10):3020-35

4. Fonarow GC et al. Timeliness of tissue-type plasminogen activator therapy in acute ischemic stroke: patient characteristics, hospital factors, and outcomes associated with door-to-needle times within 60 minutes. Circulation. 2011;123(7):7508.
5. Krumholz HM et al. Improvements in door-to-balloon time in the United States, 2005 to 2010. Circulation. 2011;124(9):1038-45.

6. Patel RD, Saver JL. Evolution of reperfusion therapies for acute brain and acute myocardial ischemia: a systematic, comparative analysis. Stroke. 2013;44(1):94-8

7. Cloft $\mathrm{HJ}$ et al. Intra-arterial stroke therapy: an assessment of demand and available work force. AJNR Am J Neuroradiol. 2009;30(3):453-8.

8. Berkhemer OA et al. A randomized trial of intraarterial treatment for acute ischemic stroke. $\mathrm{N}$ Engl J Med. 2015;372:11-20.

9. Goyal $M$ et al. Randomized assessment of rapid endovascular treatment of ischemic stroke. new england journal of medicine. 2015;372:1019-30.
10. Saver JL et al. Stent-retriever thrombectomy after intravenous t-PA vs. t-PA alone in stroke. N Engl J Med. 2015;372:2285-95.

11. Jovin TG et al. Thrombectomy within 8 hours after symptom onset in ischemic stroke. N Engl J Med. 2015;372:2296-306.

12. Topol EJ, Teirstein PS, Textbook of Interventional Cardiology (2019) 8th edition, Philadelphia: Elsevier.

13. Sevick LK et al. Systematic review of the cost and cost-effectiveness of rapid endovascular therapy for acute ischemic stroke. Stroke. 2017;48(9):2519-26.

14. Kunz W et al. Lifetime quality of life and cost consequences of treatment delays in endovascular thrombectomy for stroke based on hermes data. J Neurointerv Surg. 2018;10(Suppl 2):A1. 
15. Holmes Jr DR, Hopkins LN. Interventional cardiology and acute stroke care going forward: JACC review topic of the week. J Am Coll Cardiol. 2019;73(12):1483-90.

16. Holmes DR, Jr., Hopkins LN. Patients, practice, practicality, and politics. JACC Cardiovasc Interv. 2019;12(3):1711-3.

17. Hopkins LN, Holmes Jr DR. Public health urgency created by the success of mechanical thrombectomy studies in stroke. Circulation. 2017;135(13):1188-90.

18. Zaidat $O O$ et al. Demand-supply of neurointerventionalists for endovascular ischemic stroke therapy. Neurology. 2012;79(13 Suppl 1):S35-41.

19. Adeoye $\mathrm{O}$ et al. Geographic access to acute stroke care in the United States. Stroke. 2014;45(10):3019-24.

20. Choi JC et al. Regional availability of mechanical embolectomy for acute ischemic stroke in California, 2009 to 2010. Stroke. 2015;46(3):762-8.

21. Nikoubashman $O$ et al. Transfer of stroke patients impairs eligibility for endovascular stroke treatment. J Neuroradiol. 2018;45(1):49-53.

22. Fiorella D et al. Should neurointerventional fellowship training be suspended indefinitely? J Neurointerv Surg. 2012;4(5):315-8.

23. Slater LA et al. Too many fellows, too few jobs: the Australian reality. J Neurointerv Surg. 2016;8(e1):e17.

24. Grotta JC et al. Rethinking training and distribution of vascular neurology interventionists in the era of thrombectomy. Stroke. 2017;48(8):2313-7.

25. White $\mathrm{CJ}$ et al. Interventional stroke therapy: current state of the art and needs assessment. Catheter
Cardiovasc Interv. 2007;70(3):471-6.

26. Htyte $\mathrm{N}$ et al. Predictors of outcomes following catheter-based therapy for acute stroke. Catheter Cardiovasc Interv. 2014;85(6):1043-50.

27. Widimsky $P$ et al. Direct catheterbased thrombectomy for acute ischemic stroke: outcomes of consecutive patients treated in interventional cardiology centers in close cooperation with neurologists. $J$ Am Coll Cardiol. 2015;66(4):487-8.

28. Hornung $M$ et al. Acute stroke interventions performed by cardiologists: initial experience in a single center. JACC Cardiovasc Interv. 2019:12(17):1703-10.

29. Guidera $S$ et al. TCT-210 3 year experience with cath lab based acute stroke rescue program in a community hospital. J Am Coll Cardiol. 2018;72(Suppl 13):B88. 